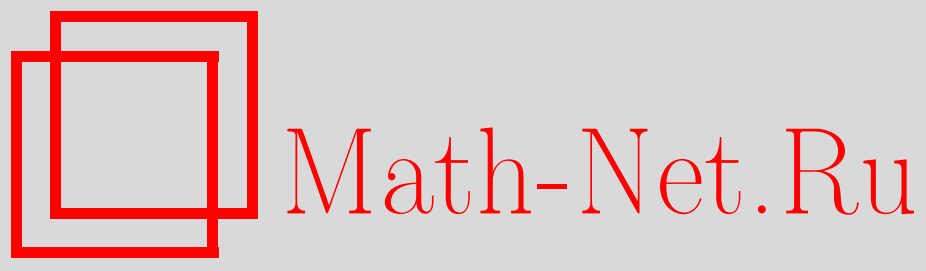

Г. М. Жислин, Спектр гамильтонианов псевдорелятивистских электронов молекул в пространствах функций перестановочной и точечной симметрии, Функи. анализ и его прил., 2006, том 40, выпуск 2, 65-69

DOI: https://doi.org/10.4213/faa7

Использование Общероссийского математического портала Math-Net.Ru подразумевает, что вы прочитали и согласны с пользовательским соглашением

http://www.mathnet.ru/rus/agreement

Параметры загрузки:

IP : 54.224 .60 .19

26 апреля 2023 г., 13:03:28

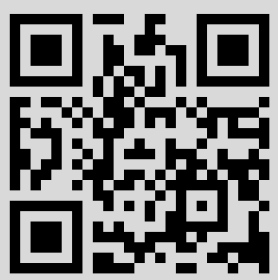




\title{
Спектр гамильтонианов псевдорелятивистских электронов молекул в пространствах функций перестановочной и точечной симметрии*
}

\author{
(c) 2006. Г. М. Жислин
}

1. Введение. В настоящей заметке мы изучаем свойства псевдорелятивистских (ПР) гамильтонианов $H_{n}$ систем $Z_{n}$, состоящих из $n$ электронов в потенциальном поле $k$ фиксированных ядер. Потенциальные части ПР операторов не релятивистские (НР), а кинетические - релятивистские. ПР операторы не локальны, и именно это порождает добавочные трудности при изучении их спектров по сравнению с НР операторами тех же систем. Несмотря на это, многие результаты, полученные ранее для НР систем $Z_{n}$ и их операторов, были доказаны позднее для ПР операторов. В частности, существенный спектр ПР операторов $H_{n}$ был найден без учета симметрии и с учетом перестановочной симметрии в [2] и с учетом вращательной - относительно групп $O^{+}(3)$ и $O(3)$ - симметрии при $k=1$ в [3]. Структура дискретного спектра оператора $H_{n}$ и спектральные асимптотики были найдены в тех же случаях для нейтральных и положительно заряженных систем $[6,5,3]$. Но для важного случая $k \geqslant 2$ (случай молекул с бесконечно тяжелыми ядрами) результаты о спектре с учетом вращательной симметрии (без спектральных асимптотик) были получены только для НР систем [4]. При $k \geqslant 2$ вращательная симметрия системы $Z_{n}$ и гамильтониана $H_{n}$ связана не с группой $O(3)$ (как при $k=1$ ), а с некоторой (главным образом, конечной) подгруппой $F_{k}$ из $O(3)$. Эта подгруппа определяется положением тождественных (друг другу) ядер; симметрия по отношению к группе $F_{k}$ называется точечной симметрией.

Ниже рассматриваются ПР операторы $H_{n}$ при любом $k \geqslant 1$ в пространствах функций, имеющих произвольные фиксированные типы перестановочной и точечной симметрии. Для этих операторов мы

(a) находим положение существенного спектра (теорема 1);

(b) получаем двусторонние оценки счетной функции дискретного спектра через счетные функции дискретного спектра некоторых двухчастичных НР операторов (теорема 3 );

(c) находим главный член спектральной асимптотики (теорема 4).

Автор искренне благодарит Международный институт математической физики им. Э. Шрёдингера (Вена) и лично проф. Ингвасона за приглашение в этот институт, где была выполнена значительная часть настоящего исследования.

2. Операторы. Пусть $Z_{n}=\{1, \ldots, n\}-$ система $n$ псевдорелятивистских электронов в потенциальном поле $k$ ядер, имеющих бесконечные массы; $e<0$ и $m$ - заряд и масса электрона; $r_{j}=\left(x_{j}, y_{j}, z_{j}\right)$ - радиус-вектор $j$-го электрона, $j=1, \ldots, n ; e_{s}>0$ и $A_{s}=\left(A_{s 1}, A_{s 2}, A_{s 3}\right)$ - заряд и радиус-вектор $s$-го ядра, $s=1, \ldots, k ; r=\left(r_{1}, \ldots, r_{n}\right), \mathbb{R}^{3 n}=\{r\}$. ПР оператор энергии системы $Z_{n}$

*Работа поддержана грантом РФФИ 03-01-715. 
может быть записан в виде

$$
\mathscr{H}_{n}=K_{n}+V_{n}(r)
$$

где

$$
\begin{gathered}
K_{n}=\sum_{i=1}^{n} K(i), \quad K(i)=\sqrt{-\Delta_{i}+m^{2}}, \quad \Delta_{i}=\frac{\partial^{2}}{\partial x_{i}^{2}}+\frac{\partial^{2}}{\partial y_{i}^{2}}+\frac{\partial^{2}}{\partial z_{i}^{2}} \\
V_{n}(r)=e^{2} \sum_{i<j ; i, j}^{1, n}\left|r_{i}-r_{j}\right|^{-1}+\sum_{i=1}^{n} \sum_{s=1}^{k} e e_{s}\left|r_{i}-A_{s}\right|^{-1} .
\end{gathered}
$$

Здесь мы выбрали систему единиц так, что постоянная Планка и скорость света равны 1. Операторы $K(i)$ не локальны в координатном пространстве; поэтому мы будем рассматривать их в импульсном представлении, где $K(i)$ превращаются в операторы умножения $\bar{K}(i)$; иными словами, фурье-образ значения $K(i) f\left(r_{i}\right)$ оператора $K(i)$ на функции $f\left(r_{i}\right)$ есть произведение $\bar{K}(i) \bar{f}\left(p_{i}\right)$, где $\bar{K}(i)=\sqrt{p_{i}^{2}+m^{2}}, \bar{f}\left(p_{i}\right)$ - фурье-образ функции $f\left(r_{i}\right), p_{i}$ - импульс $i$ го электрона. Технически удобнее вместо операторов $\bar{K}(i)$ взять операторы $\widehat{K}(i)=\bar{K}(i)-m$ и вместо $K_{n}$ - оператор $\widehat{K}_{n}=\sum_{i=1}^{n} \widehat{K}(i)$; для потенциала $V_{n}(r)$ мы сохраняем форму (1). Таким образом, вместо оператора $\mathscr{H}_{n}$ мы будем изучать оператор

$$
H_{n}=\widehat{K}_{n}+V_{n}(r) .
$$

Известно [1], что оператор $H_{n}$ ограничен снизу в $\mathscr{L}_{2}\left(\mathbb{R}^{3 n}\right)$, если

$$
e_{s}<-2 /(\pi e), \quad s=1, \ldots, k,
$$

где в выбранной системе единиц $e^{2}=137^{-1}-$ постоянная тонкой структуры, $e_{s}=-N_{s} e$, a $N_{s}$ - номер в таблице Менделеева того элемента, ядро атома которого расположено в точке $A_{s}$.

Неравенства (3) будут справедливы при

$$
N_{s} \leqslant 87, \quad s=1, \ldots, k
$$

далее мы всюду предполагаем, что неравенство (4) выполнено. Расширим (по Фридрихсу) оператор $H_{n}$ с $C_{0}^{\infty}$ до самосопряженного, сохраняя для полученного оператора обозначение $H_{n}$.

Наряду с $H_{n}$ мы будем рассматривать оператор $H_{n-1}$ системы $Z_{n-1}=$ $\{1, \ldots, n-1\}$, состоящей из $n-1$ электронов в потенциальном поле тех же ядер, что содержатся в $Z_{n}$. Этот оператор определяется аналогично оператору $H_{n}$, но в пространстве $\mathscr{L}_{2}\left(\mathbb{R}^{3 n-3}\right)$, где $\mathbb{R}^{3 n-3}=\left\{r^{\prime}\right\}, r^{\prime}=\left(r_{1}, \ldots, r_{n-1}\right)$.

3. Симметрия. Пусть $O^{+}(3)$ и $W-$ группы вращений и инверсий в $\mathbb{R}^{3}$, $O(3)=O^{+}(3) \times W$ и $F_{k}$ - такая подгруппа из $O(3)$, что преобразования $g$ из $F_{k}$ переводят тождественные ядра друг в друга. То есть для любого $s, 1 \leqslant s \leqslant k$, и $g \in F_{k}$

$$
g A_{s}=A_{t}
$$

где ядро с номером $t$ тождественно ядру с номером $s$ (т. е. $\left.e_{t}=e_{s}\right)$ и $t$ зависит от $s$ и $g$. Группа $F_{k}$ называется группой точечной симметрии системы $Z_{n}$. Пусть $S_{n}$ - группа перестановок $n$ электронов, $G_{k}=S_{n} \times F_{k}$ и $G$ есть одна из групп $F_{k}, S_{n}, G_{k}$. При $g \in G$ определим унитарные операторы $T_{g}$ формулой

$$
T_{g} \psi(r)=\psi\left(g^{-1} r\right), \quad \psi(r) \in \mathscr{L}_{2}\left(\mathbb{R}^{3 n}\right), \quad g \in G .
$$


Из (5) следует, что $T_{g} V_{n}(r)=V_{n}(r) T_{g}, g \in F_{k}$, где операторы $T_{g}$ действуют на координаты электронов. Следовательно,

$$
T_{g} H_{n}=H_{n} T_{g}, \quad g \in G_{k}=S_{n} \times F_{k} .
$$

Пусть $\alpha, \tau$ и $\sigma=(\alpha, \tau)$ суть типы неприводимых представлений групп $S_{n}, F_{k}$ и $G_{k}$ соответственно. Обозначим через $\gamma$ типы неприводимых представлений $D_{g}^{(\gamma)}$ группы $G\left(\gamma=\alpha\right.$ при $G=S_{n}, \gamma=\tau$ при $G=F_{k}, \gamma=(\alpha, \tau)$ при $\left.G=G_{k}\right)$ и через $P_{n}^{(\gamma)}$ проекторы в $\mathscr{L}_{2}\left(\mathbb{R}^{3 n}\right)$ на подпространства $B_{n}^{(\gamma)}=P_{n}^{(\gamma)} \mathscr{L}_{2}\left(\mathbb{R}^{3 n}\right)$ функций, которые преобразуются операторами $T_{g}, g \in G$, по представлениям типов $\gamma$ (выражения для $P_{n}^{(\gamma)}$ известны [9]). В силу (6) подпространства $B_{n}^{(\gamma)}$ инвариантны относительно оператора $H_{n}$. Пусть тип $\gamma$ фиксирован и $H_{n}^{(\gamma)}-$ ограничение оператора $H_{n}$ на подпространство $B_{n}^{(\gamma)}$.

Наша цель - изучение спектра оператора $H_{n}^{(\gamma)}$ для $\gamma=\sigma=(\alpha, \tau), G=G_{k}=$ $S_{n} \times F_{k}$.

Опишем перестановочную симметрию состояний системы $Z_{n-1}$. Обозначим через $\alpha^{\prime}$ типы неприводимых представлений группы $S_{n-1}$ перестановок $n-1$ электронов. Пусть $P_{n-1}^{\left(\alpha^{\prime}\right)}-$ проектор на подпространство $B_{n-1}^{\left(\alpha^{\prime}\right)}=P_{n-1}^{\left(\alpha^{\prime}\right)} \mathscr{L}_{2}\left(\mathbb{R}^{3 n-3}\right)$ функций $\varphi\left(r^{\prime}\right) \in \mathscr{L}_{2}\left(\mathbb{R}^{3 n-3}\right)$, которые преобразуются операторами $T_{g}, T_{g} \varphi\left(r^{\prime}\right)=$ $\varphi\left(g^{-1} r^{\prime}\right), g \in S_{n-1}$, по представлениям типа $\alpha^{\prime}$ группы $S_{n-1}$. Пусть $H_{n-1}^{\left(\alpha^{\prime}\right)}-$ ограничение оператора $H_{n-1}$ на $B_{n-1}^{\left(\alpha^{\prime}\right)}, E_{1}(\alpha)$ - множество всех типов $\alpha^{\prime}$, для которых представление типа $\alpha^{\prime}$ группы $S_{n-1}$ содержится в представлении типа $\alpha$ группы $S_{n}$ после сужения его с $S_{n}$ на $S_{n-1}$,

$$
B_{n-1}(\alpha)=\sum_{\alpha^{\prime} \in E_{1}(\alpha)} \oplus B_{n-1}^{\left(\alpha^{\prime}\right)}, \quad H_{n-1}(\alpha)=\sum_{\alpha^{\prime} \in E_{1}(\alpha)} H_{n-1}^{\left(\alpha^{\prime}\right)} .
$$

Оператор $H_{n-1}(\alpha)$ есть ограничение оператора $H_{n-1}$ на подпространство $B_{n-1}(\alpha)$; положим

$$
\mu_{n}^{(\alpha)}=\inf H_{n-1}(\alpha)
$$

4. Результаты о существенном спектре. Пусть группа $F_{k}$ конечна.

ТЕорема 1. Для любого $\sigma=(\alpha, \tau)$ существенный спектр $s_{\mathrm{ess}}\left(H_{n}^{(\sigma)}\right)$ операmора $H_{n}^{(\sigma)}$ coстоит из всех точек луча $\left[\mu^{(\alpha)},+\infty\right)$.

Теорема 1 означает, что для конечной группы $F_{k}$ существенный спектр оператора $H_{n}^{(\alpha, \tau)}$ не зависит от $\tau$ и совпадает с существенным спектром оператора $H_{n}^{(\alpha)}[2]$. Для бесконечных групп $F_{k}$ утверждение теоремы 1 может выполняться или не выполняться в зависимости от $F_{k}$. Так, при $F_{k}=F_{1}=O^{+}(3)$ теорема 1 верна, а при $F_{k}=F_{1}=O(3)$ - нет [3]. Поясним причины этого. Пусть $\Gamma$ - множество всех типов неприводимых представлений группы $F_{k}$, а $E_{2}(\tau)-$ множество тех $\tau^{\prime}$ из $\Gamma$, для каждого из которых существует $\tau^{\prime \prime} \in \Gamma$, такой, что

$$
D_{g}^{\left(\tau^{\prime}\right)} \otimes D_{g}^{\left(\tau^{\prime \prime}\right)} \supseteq D_{g}^{(\tau)}, \quad g \in F_{k}
$$

И

$$
\text { (a) } \quad P^{\left(\tau^{\prime}\right)} \mathscr{L}_{2}\left(\mathbb{R}^{3 n-3}\right) \neq 0, \quad \text { (b) } \quad P^{\left(\tau^{\prime \prime}\right)} \mathscr{L}_{2}\left(\mathbb{R}^{3}\right) \neq 0
$$

Пусть $E=\left\{\sigma^{\prime} \mid \sigma^{\prime}=\left(\alpha^{\prime}, \tau^{\prime}\right), \alpha^{\prime} \in E_{1}(\alpha), \tau^{\prime} \in E_{2}(\tau)\right\}$. Из доказательства теоре- 
мы 1 следует, что $\mu^{(\sigma)}:=\inf s_{\text {ess }}\left(H_{n}^{(\sigma)}\right)=\inf _{\sigma^{\prime} \in E} \inf H_{n-1}^{\left(\sigma^{\prime}\right)}$ при любых $F_{k}$. Поэтому если $E_{2}(\tau)=\Gamma$, то $\mu^{(\sigma)}=\mu^{(\alpha)}=\inf H_{n-1}(\alpha)$. В частности, при конечных $F_{k}$ и $F_{k}=O^{+}(3)$ выполняется равенство $E_{2}(\tau)=\Gamma$ [4] и теорема 1 верна. Однако при $F_{k}=O(3)$ для некоторых $\tau$ множество $E_{2}(\tau)$ не совпадает с $\Gamma$, ибо соотношение $(9(\mathrm{a}))$ не верно при $\tau^{\prime}=(0,-1)^{1)}$ и $n=3$, а соотношение $(9(\mathrm{~b}))$ не верно при $\tau^{\prime \prime}=\left(l^{\prime \prime},(-1)^{l^{\prime \prime}+1}\right), l^{\prime \prime}=0,1, \ldots$, так как четность сферических функций порядка $l$ равна $(-1)^{l}$. Потому-то при $F_{k}=O(3)$, вообще говоря, $\mu^{(\sigma)} \neq \mu^{(\alpha)}$.

5. Результаты о дискретном спектре. Пусть далее группа $F_{k}$ конечна и $Q_{n}=n e+\sum_{s=1}^{k} e_{s}$ - общий заряд системы $Z_{n}$.

Tеорема 2. Пусть $Q_{n} \geqslant 0$. Тогда число $\mu_{n}^{(\alpha)}=\inf H_{n-1}(\alpha)$ есть точка дискретного спектра оператора $H_{n-1}(\alpha)$.

Обозначим через $U$ собственное подпространство оператора $H_{n-1}(\alpha)$, отвечающее собственному значению $\mu^{(\alpha)}$. Так как $T_{g^{\prime}} H_{n-1}=H_{n-1} T_{g^{\prime}}, g^{\prime} \in G_{k}^{\prime}=$ $S_{n-1} \times F_{k}$, то пространство $U$ инвариантно относительно операторов $T_{g^{\prime}}$. Очевидно, что $g^{\prime} \rightarrow T_{g^{\prime}}$ есть представление группы $G_{k}^{\prime}$ в $U$. Для простоты предположим, что это представление неприводимо, и обозначим его тип через $\sigma_{0}^{\prime}$. Так как $G_{k}^{\prime}=S_{n-1} \times F_{k}$, то $\sigma_{0}^{\prime}=\left(\alpha_{0}^{\prime}, \tau_{0}^{\prime}\right)$ для некоторых типов $\alpha_{0}^{\prime}$ и $\tau_{0}^{\prime}$ неприводимых представлений групп $S_{n-1}$ и $F_{k}$ соответственно. Далее, для любых чисел $\varepsilon>0, R>0$ определим эффективные двухчастичные НР операторы

$$
h_{ \pm \varepsilon}(R)=\left(\frac{-1 \pm \varepsilon}{2 m}\right) \Delta_{n}+(1 \pm \varepsilon) Q_{n-1} e\left|r_{n}\right|^{-1},
$$

которые рассматриваются на гладких функциях с носителями в области $\left|r_{n}\right| \geqslant R$. Пусть $h_{ \pm \varepsilon}^{(\gamma)}(R)$ - ограничение оператора $h_{ \pm \varepsilon}(R)$ на подпространство функций точечной симметрии $\gamma$. Наконец, для любого $\lambda$ и произвольного самосопряженного оператора $B$ обозначим через $N(\lambda, B)$ счетную функцию дискретного спектра $s_{d}(B)$ оператора $B$ на полуоси $(-\infty, \lambda]$.

Tеорема 3. Пусть $Q_{n} \geqslant 0$. Тогда дискретный спектр оператора $H_{n}^{(\sigma)}$ бесконечен при любом $\sigma=(\alpha, \tau)$ и для любых $\varepsilon>0, \lambda_{0}>0$ существуют $R>0 u$ $C_{i}=C_{i}(\varepsilon, R), i=1,2$, такие, что при любом $\lambda, 0<\lambda \leqslant \lambda_{0}$,

$$
\begin{aligned}
& |\sigma| \sum_{\gamma \in \Gamma} b\left(\tau ; \tau_{0}^{\prime}, \gamma\right)|\gamma|^{-1} N\left(-\lambda, h_{-\varepsilon}^{(\gamma)}(R)\right)-C_{1} \\
& \quad \leqslant N\left(\mu_{n}^{(\alpha)}-\lambda ; H_{n}^{(\sigma)}\right) \leqslant|\sigma| \sum_{\gamma \in \Gamma} b\left(\tau ; \tau_{0}^{\prime}, \gamma\right)|\gamma|^{-1} N\left(-\lambda ; h_{+\varepsilon}^{(\gamma)}(R)\right)+C_{2},
\end{aligned}
$$

где множество Г определено в разд. $4,|\sigma|=|\alpha||\tau|,|\alpha|,|\tau|,|\gamma|$ - размерности представлений типов $\sigma, \alpha, \tau u \gamma u$

$$
b\left(\tau ; \tau_{0}^{\prime}, \gamma\right)=\left|F_{k}\right|^{-1} \sum_{g \in F_{k}} \chi_{g}^{\left(\tau_{0}^{\prime}\right)} \chi_{g}^{(\gamma)} \bar{\chi}_{g}^{(\tau)}
$$

здесъ $\left|F_{k}\right|$ - порядок группь $F_{k} u \chi_{g}^{(\delta)}$ - характер элемента $g$ в представлении muna $\delta, \delta=\gamma, \tau, \tau_{0}^{\prime}$.

1) Напомним, что типы неприводимых представлений группы $O(3)$ суть пары $(l, \omega)$, где $l(l=0,1,2, \ldots)$ и $\omega(\omega= \pm 1)$ - типы неприводимых представлений групп $O^{+}(3)$ и $W$. 
Теорема 3 дает двусторонние оценки счетной функции дискретного спектра $n$-частичного ПР оператора $H_{n}^{(\sigma)}$ через счетные функции дискретного спектра двухчастичных НР операторов (10). Используя эти оценки, мы получаем следующий итоговый результат.

ТЕОремА 4. Пусть $Q_{n} \geqslant 0$. Тогда для любого $\sigma=(\alpha, \tau)$

$$
\lim _{\lambda \rightarrow 0+0} N\left(\mu_{n}^{(\alpha)}-\lambda ; H_{n}^{(\sigma)}\right)(f(\lambda))^{-1}=|\sigma||\tau|\left|\tau_{0}^{\prime}\right|\left|F_{k}\right|^{-1},
$$

где $f(\lambda)=6^{-1} 2^{-1 / 2} m^{1 / 2}\left|Q_{n-1} e\right|^{3} \lambda^{-3 / 2}$ - главный член асимптотики счетной функции дискретного спектра ПР (и НР) оператора энергии водородоподобного иона с фиксированным ядром и зарядом $Q_{n-1}$.

6. Замечания. 1. Так как $s_{\text {ess }}\left(H_{n}^{(\alpha)}\right)=s_{\text {ess }}\left(H_{n}^{(\alpha, \tau)}\right)$ в силу теоремы 1 , то

$$
s_{d}\left(H_{n}^{(\alpha)}\right)=\bigcup_{\tau \in \Gamma} s_{d}\left(H_{n}^{(\alpha, \tau)}\right) .
$$

Равенства (13), (14) описывают внутреннюю структуру дискретного спектра $s_{d}\left(H_{n}^{(\alpha)}\right)$ по отношению к точечной симметрии. Кроме того, используя $(13),(14)$, мы можем получить главный член спектральной асимптотики для оператора $H_{n}^{(\alpha)}$ : суммируя равенства $(13)$ по $\tau$ при $\sigma=(\alpha, \tau)$ и используя первую теорему Бернсайда, получим в силу (14), что

$$
\lim _{\lambda \rightarrow 0+0} N\left(\mu_{n}^{(\alpha)}-\lambda ; H_{n}^{(\alpha)}\right)(f(\lambda))^{-1}=|\alpha|\left|\tau_{0}^{\prime}\right| .
$$

Равенство (15) согласуется с [5], если рассматривать $\left|\tau_{0}^{\prime}\right|$ как кратность неприводимого представления типа $\alpha_{0}^{\prime}$ в $U$.

2. Теорема 4 дает только главный член спектральной асимптотики аналогично $[3,5,6]$, где мы учитывали симметрию по отношению к группам $S_{n}, S_{n} \times$ $O^{+}(3), S_{n} \times O(3)$ или вообще пренебрегали симметрией. Причины те же, что в $[3,5,6]$. А именно, при использовании геометрических методов для получения второго члена спектральной асимптотики аналогично тому, как это делается [8], необходимо (как минимум) знать суммируемость в $\mathscr{L}^{2}\left(\mathbb{R}^{3 n-3}\right)$ с подходящим весом функций из $U$ (см. п. 5). K сожалению, подобные результаты есть лишь при $n=1$.

\section{ЛитерАТУрА}

1. Lieb E., Yau H.-T. Comm. Math. Phys., 118, 177-213 (1988). 2. Lewis R. T., Siedentop H., Vugalter S. Ann. Inst. H. Poincaré Phys. Théor. 67, No. 1, 1-28 (1997). 3. Жислин Г. М. Докл. РАН, 397, №1 (2004). 4. Жислин Г. М., Манделъ Е. Л. Теор. матем. физ., 1, № 2, 295-301 (1969). 5. ЖКислин Г. М. Препринт НИРФИ № 473, Н. Новгород, 2002. 6. Жислин Г. М., Вугалътер C. А. Изв. РАН, сер. матем., 66, №1, 71-102 (2002). 7. Вугальтер С. А., Жислин Г. М. Теор. матем. физ., 21, № 2, 297-306 (1999). 8. Вугальтер С. А., Жислин Г. М. Труды ММО, 54, 187-213 (1991). 9. Вигнер Е. Теория групп. ИЛ, М., 1961.

Нижегородский научно-исследовательский радиофизический институт 